\title{
Review Article \\ Impact of Residual Fragments following Endourological Treatments in Renal Stones
}

\author{
Cenk Acar ${ }^{1}$ and $\mathrm{Cag} \mathrm{Cal}^{2}$ \\ ${ }^{1}$ Department of Urology, Faculty of Medicine, Pamukkale University, 20070 Denizli, Turkey \\ ${ }^{2}$ Department of Urology, Faculty of Medicine, Ege University, 35100 Izmir, Turkey
}

Correspondence should be addressed to Cag Cal, cagcal1@cagcal.com

Received 27 February 2012; Revised 30 May 2012; Accepted 8 June 2012

Academic Editor: M. Hammad Ather

Copyright ( 92012 C. Acar and C. Cal. This is an open access article distributed under the Creative Commons Attribution License, which permits unrestricted use, distribution, and reproduction in any medium, provided the original work is properly cited.

\begin{abstract}
Today, shock wave lithotripsy (SWL), percutaneous nephrolithotomy (PCNL), and flexible ureterorenoscopy (URS) are the most widely used modalities for the management of renal stones. In earlier series, treatment success of renal calculi assessed with KUB radiography, ultrasound, or intravenous pyelography which are less sensitive than CT that leads to be diversity of study results in reporting outcome. Residual fragments (RFs) after interventional therapies may cause pain, infection, or obstruction. The size and location of RFs following SWL and PCNL are the major predictors for clinical significant symptoms and stone events requiring intervention. There is no consensus regarding schedule for followup of SWL, PCNL, and flexible URS. Active monitoring can be recommended when the stones become symptomatic, increase in size, or need intervention. RFs $<4 \mathrm{~mm}$ after $S W L$ and $<2 \mathrm{~mm}$ after PCNL and flexible URS could be actively monitored on an annual basis with CT. Early repeat SWL and second-look endoscopy are recommended after primary SWL and PCNL, respectively. There is insufficient data for flexible URS, but RFs can be easily treated with repeat URS. Finally, medical therapy should be tailored based on the stone analysis and metabolic workup that may be helpful to prevent regrowth of the RFs.
\end{abstract}

\section{Introduction}

Urolithiasis is a common disorder, affecting $3 \%$ to $5 \%$ of the population in industrialized countries and gradually increasing all around the world $[1,2]$. Today, shock wave lithotripsy (SWL), percutaneous nephrolithotomy (PCNL), and flexible ureteroscopy (URS) are widely used for renal stones. The overall success rates of SWL, PCNL, and URS in renal stones are $13.6-91.2 \%$ [3-5], 40-90\% [6], and 34-92.2\% [7, 8] in different studies, respectively. The success of these treatments also accepted free-of-stone rates in a radiologic studies such as kidney ureter bladder (KUB) radiography, ultrasonography (US), and abdominal computerized tomography (CT). CT is certainly the most sensitive method for assessing residual stones. Although CT detects stone fragments down to $1 \mathrm{~mm}$ in all subtypes of stones (radiolucent uric acid or cystine calculi as well as calcium-containing stones), the clinical value of being able to detect very small fragments remains debatable [9]. Besides, detecting negligible RF with $\mathrm{CT}$ is time-consuming and expensive. Although the renal stone protocol on newer multidetector scanners has been widely used, they lead to expose 25-50-fold higher doses of radiation than KUB radiography [10]. The advantages of ultrasound are providing comparable precision and detecting stones down to a diameter of $2 \mathrm{~mm}$ [11]. The clinicians suggested that evaluation of asymptomatic patients after SWL could be limited to routine radiologic studies such as KUB radiography and US [11-14].

Clinically insignificant residual stone fragments (CIRF) defined as residual calculi $<4$ or $5 \mathrm{~mm}$ while the patient is asymptomatic and the stone composition is not struvite or infection stone [15-17]. Residual fragments (RF) may cause pain, infection, or obstruction whether clinically significant or not. These patients could need auxiliary measures, including further SWL sessions, double-J stent insertions, or endoscopic surgical treatment. In addition, RFs are important that may act as nidus for regrowth. At this point of view, term of CIRF reflects a common and still controversial problem for SWL, PCNL, or URS.

To define follow-up protocols and timing of auxiliary intervention, better understanding of the natural history 
of RFs following endourological treatments in renal stones should be needed. In this paper, we discussed fate of RF following SWL, PCNL, and flexible URS in kidney stones with recent literature.

\section{Residual Fragments following SWL}

In recent literature, several studies evaluated the fate of RFs after SWL $[11-14,18]$. Spontaneous stone passage was found in $13.6 \%$ to $51.3 \%$ of the patients with mean followup between 15 and 57 months. Stone size remained stable in $17.3 \%$ to $52.6 \%$ of the patients, while stone regrowth was encountered in $21.4 \%$ to $69 \%$. El-Nahas et al. reported that $52(33.7 \%)$ of 154 patients had significant clinical stone event that need further intervention (mostly SWL) [18]. Osman et al. noted that $21.4 \%$ of the residual stone led to stone recurrence and need for retreatment [14]. The spontaneous clearance rate was affected by location of RF. The highest rates are seen in the ureter. In addition, many investigators emphasized that RFs are commonly localized to lower-pole calyces after SWL wherever treated in the kidney [5]. In a review, the authors concluded that $25 \%$ of the patients with CIRF will become stone-free, $50 \%$ will remain symptom-free, $20 \%$ will experience a clinically significant stone episode, and $4 \%$ to $25 \%$ of patients will need a secondary intervention, especially SWL [16]. According to this prediction, auxiliary endoscopic intervention should be considered as overtreatment in asymptomatic patients [16]. In a recent systematic review, the natural history and clinical significance of small and asymptomatic RFs after SWL were extensively discussed with data of four studies. Totally 463 patients with CIRF ( $<5 \mathrm{~mm}$ in diameter) were followed with a period of 15 months to 4.9 years. The stone-free rates were $23.8 \%$ to $78.9 \%$ and $10.7 \%$ to $41.9 \%$ of the stones remained stable. The stone size increased in $2 \%$ to $58.6 \%$ of the patients. Between $41.4 \%$ and $100 \%$ of the patients were asymptomatic while up to $58.6 \%$ had a symptomatic episode or needed intervention with a mean followup at 15 to 57 months after SWL [19].

Most investigators indicated that the risk factors of CIRF becoming clinically significant are stone burden and number and location of RF $[14,18]$. The rate of complications and number of auxiliary interventions increased during long follow-up period [11]. El-Nahas et al. noted that patients with previous stone disease had 4.26 times higher incidence for stone events [18]. In a prospective study, the authors could not find any relationship between metaphylaxis and stone regrowth [14]. The results of these studies reflect that significant numbers of patients will need auxiliary intervention or be symptomatic during followup of SWL treatment. Consequently, the patients with RF should be actively monitored whether clinically significant or not.

On the other hand, in a prospective randomized comparative study, the author suggested that SWL retreatment promotes discharge of persistent caliceal RF after primary SWL [20]. However, it is taking into consideration the potentially higher side effects and additional cost against the advantages of a higher stone-free rate by SWL retreatment.
As a conclusion, there is no consensus regarding schedule for followup. Active monitoring can be recommended when the stones become symptomatic, increase in size, or need intervention. Most studies reported that the clearance rate is higher at 6 months, but spontaneous clearance may last up to 24 months $[11,16,18]$. In standard followup, the authors recommended clinical examination, urine culture, and routine radiographic studies as KUB radiography and/or renal US at 3-, 6-, or 12-month intervals. Such an intense followup may not be indicated for all patients because spontaneous stone clearance asymptomatically occurs in most of the patients. Therefore, any auxiliary procedures are rarely required. We recommend the initial followup can be performed at 6 months and it can be followed by an annual schedule for the patients who were not clear at that time. The intervention should be relatively noninvasive and consisted of either repeated SWL or retrograde endoscopy.

\section{Residual Fragments following PCNL}

Percutaneous nephrolithotomy is an effective minimally invasive procedure that is preformed for treatment of large and complex calculi [21]. Altunrende et al. investigated the medium-term outcome of CIRFs. They also measured 24-hour urine metabolic analysis. After PCNL, 38 patients with CIRFs followed periodically in a median time of 28.4 months. Ten $(26.3 \%)$ patients had symptomatic episode that need medical therapy. The RF size was stable or decreased in $27(71.1 \%)$ cases whilst increased in $8(21.1 \%)$ patients. Spontaneous stone rate was $7.9 \%$ in medium term of followup. They concluded that the progression could be seen in 2-year followup, whereas presence of risk factors on 24hour urine metabolic analysis could not predict growth of RFs [22]. Ganpule and Desai retrospectively examined in 187 patients to evaluate the fate of residual stones after PCNL [23]. $57.7 \%$ of RF was located in the lower calix, and the mean size of RF was $38.6 \pm 52 \mathrm{~mm}^{2}$. Fourty-five percent of the patients were stone-free without intervention at a mean followup of 24 months. Most of the stones $(65.47 \%)$ spontaneously passed in 3 months. Small RFs $\left(<25 \mathrm{~mm}^{2}\right)$ in renal pelvis had best clearance rate. In addition, a history of previous intervention, renal failure, metabolic abnormalities such as hyperuraecemia and hypercalciuria, size of RF, and experience of surgeon affected the fate of RF after PCNL in multivariate analysis. Raman et al. prospectively evaluated 42 patients with RF after PCNL with a median followup of 41 months [24]. They performed abdominal CT to determine the RF and classify the RF by using cut-off size as $2 \mathrm{~mm}$. Sixty percent of RF was $2 \mathrm{~mm}$ or smaller and $79 \%$ was $<5 \mathrm{~mm}$. The location of RF was $47 \%$ lower, $32 \%$ middle, $24 \%$ upper pole, and $18 \%$ renal pelvis/ureter. Fortythree percent of patients experienced a stone-related event with a median time to occurrence at 32 months. Of these patients, 61\% underwent auxiliary procedures such as URS, PCNL, SWL, nephrectomy, and double-J stent placement, but mostly URS. They calculated the estimated 3- and 5year event-free probabilities were $74 \%$ and $48 \%$, respectively. On multivariate analysis, a maximum $\mathrm{RF}$ size $>2 \mathrm{~mm}$ and 
location in the renal pelvis or ureter independently predicted a stone-related event. The probability of needing a second surgical procedure correlated with RF size. Eight percent of patients with RFs $<2 \mathrm{~mm}$ underwent surgical retreatment compared with $53 \%$ of patients with RFs $>2 \mathrm{~mm}$. These results indicate that patients with RFs $>2 \mathrm{~mm}$ or fragments located in the renal pelvis or ureter should be treated immediately. On the other hand, some authors evaluated cost comparison of immediate second-look endoscopy against surveillance in post-PCNL RFs. They concluded that secondlook endoscopy is cost advantageous in the patients with RF $>4 \mathrm{~mm}$ following PCNL which generally leads to stone event [25].

The schedule of followup following PCNL is still controversial. Taking into consideration that the majority of these stones will pass spontaneously without causing any symptoms during the first year of followup, an annual followup with a CT scan could be sufficient [19].

\section{Residual Fragments following Flexible URS}

Owing to technological advances, flexible URS has expanded its indications to include the stones in kidney [26]. The indication of flexible URS for renal stones is the stones less than $15 \mathrm{~mm}$ which do not respond to SWL [27]. The one of the advantages of flexible URS is the possibility of treating renal and ureteral stones in the same patient in a single session. In early series, treatment success of renal calculi was assessed with KUB radiography, US, or intravenous pyelography which are less sensitive than $\mathrm{CT}$ that leads to be diversity of study results. Breda et al. evaluated 51 patients with 161 renal stones (mean stone size of $6.6 \mathrm{~mm}$ ) and they found the overall stone-free rate after single and second procedures was $64.7 \%$ and $92.2 \%$, respectively [8]. In a different study, Perlmutter et al. evaluated the impact of stone location on success rates of flexible URS [28]. A total of 86 renal stones were treated, and the stone-free rates for upper-, middle-, and lower-caliceal stones were $100 \%, 95.8 \%$, and $90.9 \%$, respectively. They concluded that stone location does not significantly affect stone clearance rates. In a prospective randomized trial, Pearle et al. compared SWL (32 patients) and URS (35 patients) for lower-pole caliceal stones of $1 \mathrm{~cm}$ or less [29]. The stone-free rates of SWL and flexible URS were $35 \%$ and $50 \%$, respectively. They concluded that there was a statistically insignificant difference in stone-free rates between SWL and URS for the treatment of small lowerpole renal calculi. However, SWL was associated with greater patient acceptance and shorter convalescence. Tanriverdi et al. evaluated the stone-free rate of flexible URS in renal calculi by classifying the RFs as the largest single fragment $<2 \mathrm{~mm},<4 \mathrm{~mm}$, and $>4 \mathrm{~mm}$ in CT [30]. The success rates were $50.4 \%, 62.8 \%$, and $84.1 \%$ in stone-free, CIRF $<2 \mathrm{~mm}$ and CIRF $<4 \mathrm{~mm}$, respectively.

According to aforementioned studies, all RFs may be regrowth and become symptomatic regardless of stone size $[13,24,30]$. In a recent study, Rebuck et al. evaluated the fate of RFs following flexible URS in 51 patients with renal calculi [31]. Although the follow-up protocol was complex, patients with RFs actively monitored with abdominal CT and term of CIRF $(\leq 4 \mathrm{~mm})$ was used for RFs. They excluded patients who had fragments in ureter after URS and patients with complex renal anatomy. Mean follow-up duration was 18.9 months. During the follow-up period, $19.6 \%$ of the patients experienced at least one stone event, $21.7 \%$ spontaneously passed their fragments, and $58.7 \%$ remained asymptomatic over time. The stone event occurs in mean followup of 26.8 months. Most of the patients with stone event treated with repeated URS. Among the patients with spontaneous passage, $60 \%$ passed their fragments after a mean followup of 9.1 months. In contrast to several studies, stone regrowth rate was very low in this study. In addition, the authors noted that stone events occur rather than lowerpole RF location while most of remaining stones located in lower pole. They attributed these results to small sample size, compliance of their patients against stone prevention therapy, and short followup of patients with asymptomatic RFs. As a conclusion, approximately one in five patients will experience stone events over 1.6 years after flexible URS and most of them can easily be treated with URS. Although, there is no consensus on the schedule of followup, asymptomatic patients with RF can actively monitor with abdominal CT until patients become symptomatic or 2 years of followup.

\section{Medical Management on RF following Endourological Procedures}

Several authors discussed medical therapy in the management of RFs after SWL or PCNL [32-36]. Fine et al. evaluated 80 patients to determine the effect of medical therapy in a retrospective study. Patients who received medical therapy had significant decrease in the stone-formation rate from a median of 1.17 to 0.00 stones per patient per year while the patients who did not receive medical therapy had a minimal decrease from a median of 1.33 to 0.77 stones per patient per year. The medically treated patients had a significantly greater stone remission rate $(63.9 \%$ versus $23.1 \%)$ and lower stone burden increase $(27.8 \%$ versus $61.6 \%)$ than the untreated patients. Regarding patients with CIRF, $16 \%$ of medically treated patients had fragment regrowth compared with $54.5 \%$ of the untreated patients. The authors concluded that medical therapy may alleviate stone regrowth in patients with residual stone fragments after SWL [33]. Cicerello et al. evaluated the effect of alkaline citrate therapy in patients with $\mathrm{RF}<5 \mathrm{~mm}$ [32]. In up to $74 \%$ of calcium oxalate patients and in up to $86 \%$ of infection stone patients, the RFs became undetectable in 1 year of followup. The undetectable RF rates of the patients were $32 \%$ and $40 \%$ in the control group for calcium oxalate and infection stones, respectively. RF regrowth rate decreased $47 \%$ to $5 \%$ in calcium oxalate patients with medical treatment. No significant difference was found for infection stones [32]. Similarly, Soygür et al. reported that among the patients with lower-pole RFs after SWL, the patients who were receiving citrate therapy showed a significantly higher stone fragment-disappearance rate ( $45.5 \%$ versus $12.5 \%)$ and less stone recurrence rate $(56.6 \%$ versus $87.5 \%$ ) compared with the control group at 12 months 
of followup [36]. Kang et al. evaluated the effect of medical therapy management in patients with RFs following PCNL. Patients receiving medical therapy had a lower median stonefree rate ( 0.02 versus 1.00 stones per patient per year) and a higher remission rate (77\% versus $21 \%$ ) compared with patients not receiving medical therapy. This result indicated that medical therapy can inhibit new stone formation or growth in patients with residual fragments after PCNL [34]. Afterwards, there is little evidence related to effectiveness of medical therapy in patients with RFs after flexible URS. Rebuck et al. speculated the high rate of stones remaining stable was related to medical therapy strategy in particularly lower-pole location [31].

There is no valuable data for determining the cost effectiveness of medical therapy after interventional therapies. However, the cost analysis of interventional therapies may help to make better estimation for medical management. Few studies have evaluated the cost of different treatment strategies for treatment of renal calculi. Koo et al. compared the cost effectiveness and outcome of SWL and flexible URS for lower-pole renal calculi $\leq 20 \mathrm{~mm}$ [37]. The costs of flexible URS and SWL for each session were calculated approximately $\$ 4,116$ and $\$ 673$, respectively. In another study, comparing clinical outcomes and the estimated cost of PCNL and flexible URS for 2 to $3 \mathrm{~cm}$ renal stones, the estimated cost of PCNL was significantly greater than flexible URS ( $\$ 19,845$ versus $\$ 6,675$, resp.) [38]. Regarding to determine the cost effectiveness of medical therapy and prophylaxis, Lotan et al. published an international comparison for costs of medical treatments [39]. The authors classified the treatments into 3 categories as conservative therapy (comprised dietary modification without drug treatment or metabolic evaluation), empiric medical therapy (both drug and dietary treatment were initiated without metabolic evaluation in all patients), and directed medical therapy (based on comprehensive metabolic evaluation). The study result showed that conservative therapy was the least costly strategy in all countries except the UK, but was associated with the highest stone recurrence rate $(0.3$ stones/patient/year) for recurrent stone formers. The empiric therapy amounted $\$ 508$ in America, $\$ 150$ in Turkey, and $\$ 29$ in UK per patient in one year. In other countries with relatively lowmedication costs, only small differences between empiric and conservative therapy were demonstrated. Drug therapy (empiric and directed) was associated with a significantly lower rate of stone recurrence than conservative therapy. The authors emphasized that empiric therapy was minimally more effective than directed therapy because of effectiveness of medication in all treated patients whether they had a metabolic abnormality or not. Thus, medical treatment after interventional therapy seems to be more cost effective than auxiliary treatments for RFs. As a conclusion, medical therapy after endourological treatment should not replace the appropriate interventional therapy that the best chance to achieve a stone-free status with minimal morbidity. However, metabolic workup should immediately be performed before stone therapy. It may help to diagnose underlying metabolic abnormality and medical therapy may be begun promptly.

\section{Summary}

The size and location of RFs following SWL and PCNL therapies are the major predictors for clinical significant symptoms and stone events which requiring intervention. RFs $<4 \mathrm{~mm}$ after SWL and $<2 \mathrm{~mm}$ after PCNL and flexible URS could be actively monitored on an annual basis with CT. Early repeat SWL and second-look endoscopy are recommended after primary SWL and PCNL, respectively. There is no sufficient data for flexible URS but RFs can be easily treated with repeat URS. Finally, medical therapy according stone analysis and metabolic workup might be helpful to prevent regrowth of the RFs.

\section{References}

[1] K. K. Stamatelou, M. E. Francis, C. A. Jones, L. M. Nyberg, and G. C. Curhan, "Time trends in reported prevalence of kidney stones in the United States: 1976-1994," Kidney International, vol. 63, no. 5, pp. 1817-1823, 2003.

[2] A. Hesse, E. Brändle, D. Wilbert, K. U. Köhrmann, and P. Alken, "Study on the prevalence and incidence of urolithiasis in Germany comparing the years 1979 vs. 2000," European Urology, vol. 44, no. 6, pp. 709-713, 2003.

[3] C. Obek, B. Onal, K. Kantay et al., "The efficacy of extracorporeal shock wave lithotripsy for isolated lower pole calculi compared with isolated middle and upper caliceal calculi," Journal of Urology, vol. 166, no. 6, pp. 2081-2085, 2001.

[4] B. Turna, F. Ekren, O. Nazli et al., "Comparative results of shockwave lithotripsy for renal calculi in upper, middle, and lower calices," Journal of Endourology, vol. 21, no. 9, pp. 951956, 2007.

[5] S. Sozen, B. Kupeli, C. Acar, S. Gurocak, U. Karaoglan, and I. Bozkirli, "Significance of lower-pole pelvicaliceal anatomy on stone clearance after shockwave lithotripsy in nonobstructive isolated renal pelvic stones," Journal of Endourology, vol. 22, no. 5, pp. 877-881, 2008.

[6] J. Park, B. Hong, T. Park, and K. P. Hyung, "Effectiveness of noncontrast computed tomography in evaluation of residual stones after percutaneous nephrolithotomy," Journal of Endourology, vol. 21, no. 7, pp. 684-687, 2007.

[7] M. A. Ben Saddik, S. Al-Qahtani Sejiny, M. Ndoye et al., "Flexible ureteroscopy in the treatment of kidney stone between 2 and $3 \mathrm{~cm}$," Progres en Urologie, vol. 21, no. 5, pp. 327-332, 2011.

[8] A. Breda, O. Ogunyemi, J. T. Leppert, and P. G. Schulam, "Flexible ureteroscopy and laser lithotripsy for multiple unilateral intrarenal stones," European Urology, vol. 55, no. 5, pp. 1190-1197, 2009.

[9] C. Türk, T. Knoll, A. Petrik, K. Sarica, M. Straub, and C. S. Residual Stones, EAU Guidelines of Urolithiasis, vol. 61, European Association of Urology (EAU), Arnhem, The Netherlands, 2011.

[10] V. M. Pais Jr, A. L. Payton, and C. A. LaGrange, "Urolithiasis in pregnancy," Urologic Clinics of North America, vol. 34, no. 1, pp. 43-52, 2007.

[11] A. Khaitan, N. P. Gupta, A. K. Hemal, P. N. Dogra, A. Seth, and M. Aron, "Post-ESWL, clinically insignificant residual stones: reality or myth?” Urology, vol. 59, no. 1, pp. 20-24, 2002.

[12] K. Afshar, G. McLorie, F. Papanikolaou et al., "Outcome of small residual stone fragments following shock wave lithotripsy in children," Journal of Urology, vol. 172, no. 4, pp. 1600-1603, 2004. 
[13] C. Candau, C. Saussine, H. Lang, C. Roy, F. Faure, and D. Jacqmin, "Natural history of residual renal stone fragments after ESWL," European Urology, vol. 37, no. 1, pp. 18-22, 2000.

[14] M. M. Osman, Y. Alfano, S. Kamp et al., "5-Year-follow-up of patients with clinically insignificant residual fragments after extracorporeal shockwave lithotripsy," European Urology, vol. 47, no. 6, pp. 860-864, 2005.

[15] F. C. Delvecchio and G. M. Preminger, "Management of residual stones," Urologic Clinics of North America, vol. 27, no. 2, pp. 347-354, 2000.

[16] J. J. Rassweiler, C. Renner, C. Chaussy, and S. Thüroff, "Treatment of renal stones by extracorporeal shockwave lithotripsy. An update," European Urology, vol. 39, no. 2, pp. 187-199, 2001.

[17] Y. H. Tan and M. Wong, "How significant are clinically insignificant residual fragments following lithotripsy?" Current Opinion in Urology, vol. 15, no. 2, pp. 127-131, 2005.

[18] A. R. El-Nahas, A. M. El-Assmy, K. Madbouly, and K. Z. Sheir, "Predictors of clinical significance of residual fragments after extracorporeal shockwave lithotripsy for renal stones," Journal of Endourology, vol. 20, no. 11, pp. 870-874, 2006.

[19] A. Skolarikos, M. P. Laguna, G. Alivizatos, A. R. Kural, and J. J. M. C. H. De La Rosette, "The role for active monitoring in urinary stones: a systematic review," Journal of Endourology, vol. 24, no. 6, pp. 923-930, 2010.

[20] F. Krings, C. Tuerk, I. Steinkogler, M. Marberger, D. M. Newman, and L. P. Sonda, "Extracorporeal shock wave lithotripsy retreatment ("stir-up") promotes discharge of persistent caliceal stone fragments after primary extracorporeal shock wave lithotripsy," Journal of Urology, vol. 148, no. 3, pp. 1040-1042, 1992.

[21] S. C. Kim, R. L. Kuo, and J. E. Lingeman, "Percutaneous nephrolithotomy: an update," Current Opinion in Urology, vol. 13, no. 3, pp. 235-241, 2003.

[22] F. Altunrende, A. Tefekli, R. J. Stein et al., "Clinically insignificant residual fragments after percutaneous nephrolithotomy: medium-term follow-up," Journal of Endourology, vol. 25, no. 6, pp. 941-945, 2011.

[23] A. Ganpule and M. Desai, "Fate of residual stones after percutaneous nephrolithotomy: a critical analysis," Journal of Endourology, vol. 23, no. 3, pp. 399-403, 2009.

[24] J. D. Raman, A. Bagrodia, A. Gupta et al., "Natural history of residual fragments following percutaneous nephrostolithotomy," Journal of Urology, vol. 181, no. 3, pp. 1163-1168, 2009.

[25] J. D. Raman, A. Bagrodia, K. Bensalah, M. S. Pearle, and Y. Lotan, "Residual fragments after percutaneous nephrolithotomy: cost comparison of immediate second look flexible nephroscopy versus expectant management," Journal of Urology, vol. 183, no. 1, pp. 188-193, 2010.

[26] D. J. Galvin and M. S. Pearle, "The contemporary management of renal and ureteric calculi," BJU International, vol. 98, no. 6, pp. 1283-1288, 2006.

[27] M. Y. C. Wong, "Flexible ureteroscopy is the ideal choice to manage a $1.5 \mathrm{~cm}$ diameter lower-pole stone," Journal of Endourology, vol. 22, no. 9, pp. 1845-1859, 2008.

[28] A. E. Perlmutter, C. Talug, W. F. Tarry, S. Zaslau, H. Mohseni, and S. J. Kandzari, "Impact of stone location on success rates of endoscopic lithotripsy for nephrolithiasis," Urology, vol. 71, no. 2, pp. 214-217, 2008.

[29] M. S. Pearle, J. E. Lingeman, R. Leveillee et al., "Prospective randomized trial comparing shock wave lithotripsy and ureteroscopy for lower pole caliceal calculi $1 \mathrm{~cm}$ or less," Journal of Urology, vol. 179, no. 5, pp. S69-S73, 2008.
[30] O. Tanriverdi, M. S. Silay, and C. Miroglu, "Computed tomography-determined stone-free rates for ureteroscopy of upper tract stones," Journal of Endourology, vol. 23, no. 11, pp. 379-382, 2009.

[31] D. A. Rebuck, A. MacEjko, V. Bhalani, P. Ramos, and R. B. Nadler, "The natural history of renal stone fragments following ureteroscopy," Urology, vol. 77, no. 3, pp. 564-568, 2011.

[32] E. Cicerello, F. Merlo, G. Gambaro et al., "Effect of alkaline citrate therapy on clearance of residual renal stone fragments after extracorporeal shock wave lithotripsy in sterile calcium and infection nephrolithiasis patients," Journal of Urology, vol. 151, no. 1, pp. 5-9, 1994.

[33] J. K. Fine, C. Y. C. Pak, G. M. Preminger, J. W. Segura, and M. Marberger, "Effect of medical management and residual fragments on recurrent stone formation following shock wave lithotripsy," Journal of Urology, vol. 153, no. 1, pp. 27-33, 1995.

[34] D. E. Kang, M. M. Maloney, G. E. Haleblian et al., "Effect of medical management on recurrent stone formation following percutaneous nephrolithotomy," Journal of Urology, vol. 177, no. 5, pp. 1785-1789, 2007.

[35] K. Sarica, S. Erturhan, C. Yurtseven, and F. Yağci, "Effect of potassium citrate therapy on stone recurrence and regrowth after extracorporeal shockwave lithotripsy in children," Journal of Endourology, vol. 20, no. 11, pp. 875-879, 2006.

[36] T. Soygür, A. Akbay, and S. Küpeli, "Effect of potassium citrate therapy on stone recurrence and residual fragments after shockwave lithotripsy in lower caliceal calcium oxalate urolithiasis: a randomized controlled trial," Journal of Endourology, vol. 16, no. 3, pp. 149-152, 2002.

[37] V. Koo, M. Young, T. Thompson, and B. Duggan, "Costeffectiveness and efficiency of shockwave lithotripsy vs flexible ureteroscopic holmium:yttrium-aluminium-garnet laser lithotripsy in the treatment of lower pole renal calculi," BJU International, 2011.

[38] E. S. Hyams and O. Shah, "Percutaneous nephrostolithotomy versus flexible ureteroscopy/holmium laser lithotripsy: cost and outcome analysis," Journal of Urology, vol. 182, no. 3, pp. 1012-1017, 2009.

[39] Y. Lotan, J. A. Cadeddu, and M. S. Pearle, "International comparison of cost effectiveness of medical management strategies for nephrolithiasis," Urological Research, vol. 33, no. 3, pp. 223-230, 2005. 


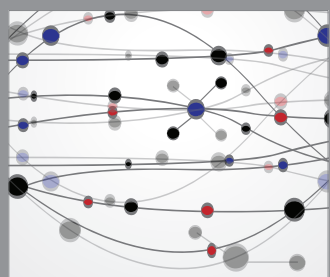

The Scientific World Journal
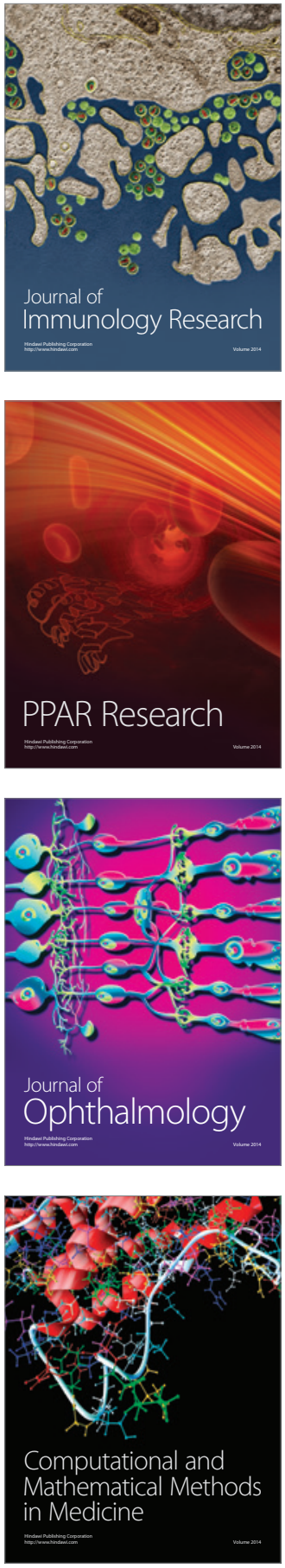

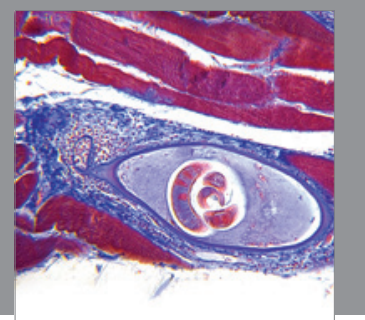

Gastroenterology

Research and Practice
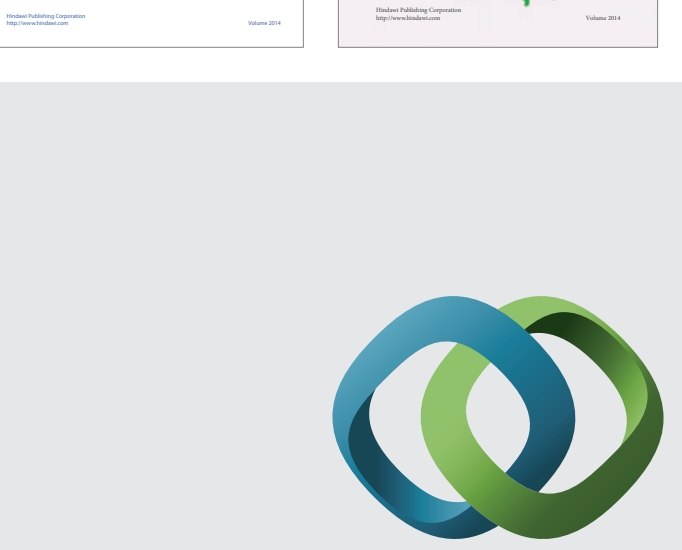

\section{Hindawi}

Submit your manuscripts at

http://www.hindawi.com
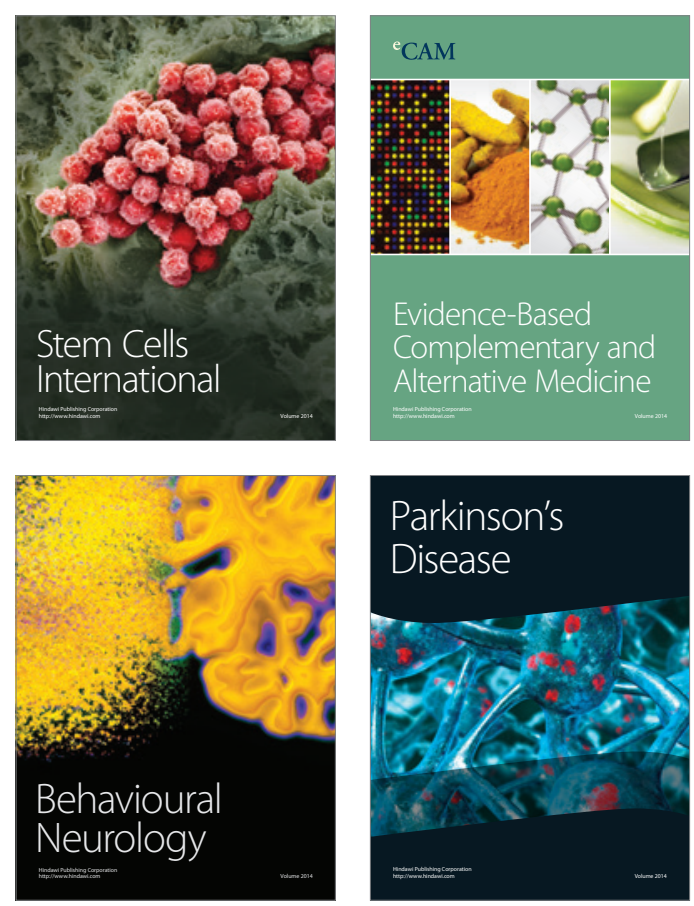

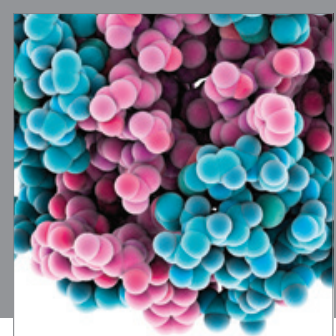

Journal of
Diabetes Research

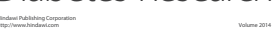

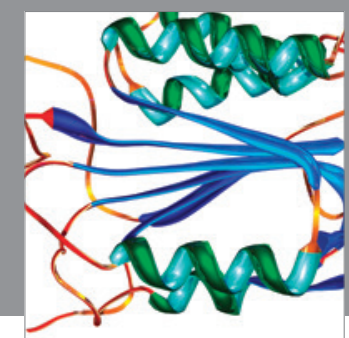

Disease Markers
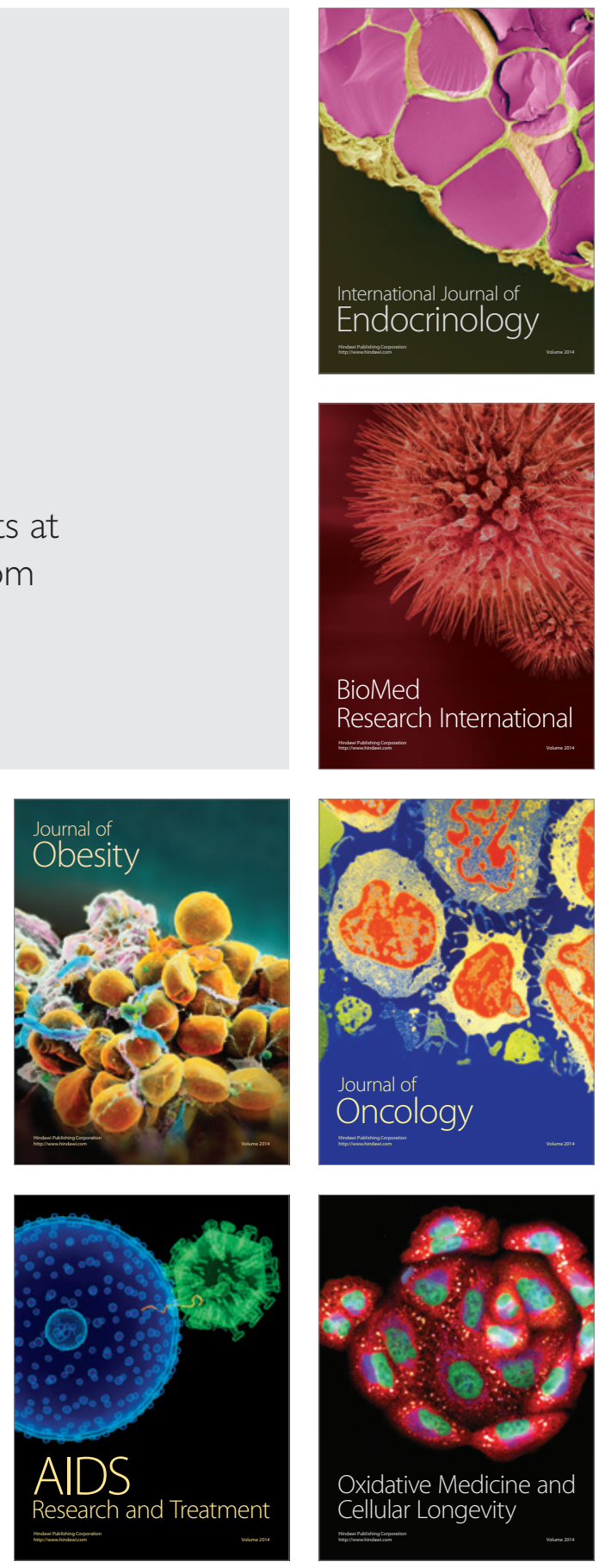